Article

\title{
Comparative Analysis of Different Model-Based Controllers Using Active Vehicle Suspension System
}

\author{
Yumna Shahid and Minxiang Wei * \\ College of Energy and Power Engineering, Nanjing University of Aeronautics and Astronautics, \\ Nanjing 210016, China; yumnashahid93@gmail.com \\ * Correspondence: weimx@nuaa.edu.cn
}

Received: 27 October 2019; Accepted: 2 December 2019; Published: 26 December 2019

check for updates

\begin{abstract}
This paper deals with the active vibration control of a quarter-vehicle suspension system. Damping control methods investigated in this paper are: higher-order sliding mode control (HOSMC) based on super twisting algorithm (STA), first-order sliding mode control (FOSMC), integral sliding mode control (ISMC), proportional integral derivative (PID), linear quadratic regulator (LQR) and passive suspension system. Performance comparison of different active controllers are analyzed in terms of vertical displacement, suspension travel and wheel deflection. The theoretical, quantitative and qualitative analysis verify that the STA-based HOSMC exhibits better performance as well as negate the undesired disturbances with respect to FOSMC, ISMC, PID, LQR and passive suspension system. Furthermore, it is also robust to intrinsic bounded uncertain dynamics of the model.
\end{abstract}

Keywords: higher-order sliding mode control; quarter-car; super twisting algorithm; active suspension system

\section{Introduction}

Suspension systems are considered important in terms of providing ride ease to passengers, capability of handling road disturbances, and stabilizing constancy of vehicles. Immense attention has been given to vehicle suspension systems in the automobile industry. Suspension systems aim to improve three main contradictory indices that are: suspension deflection, ride ease, and road disturbances [1]. Furthermore, in order to control vehicle body vibration, suspension systems should be capable of adjusting the system energy, retaining the tyre grip and reducing the effect of external disturbances such as braking and steering in this way ride comfort can be enhanced [2,3].

There are three fundamental kinds of damping suspension systems: passive, semi-active and active. Passive suspension is the least complex system and has numerous advantages. But, the disadvantage of passive suspension is the limit of overcoming unwanted oscillations that occurs due to rough road surfaces [4]. Under normal parameters, passive performance is confined as it includes fixed spring and damper. Similarly, its efficiency depend on the fixed standard of certain automobile parameters. Thus, to achieve better performance results, semi-active suspension systems using variable damper are introduced. In contrast to passive, semi-active damping system needs considerably less energy, and it is additionally non-complicated and convenient system. Therefore, it can provide significant progress in ride performance. Karnopp presented skyhook control methodology, which is widely used control approach for semi-active suspension systems as it can decrease the 'resonant peak' of vehicles and can help in achieving better ride performance [5]. In semi-active, magneto-rheological dampers (MR) are one of the most studied approach due to their low power consumption and protection specifications [6], but they only disintegrates energy and if the power fails, they act as passive suspension systems so their performance are also limited. Therefore, active suspension systems using hydraulic, air, or electric power actuator are introduced. Active suspension system composes of power source which generates desired force. Also, it does not consume energy while producing forces which avoid suspension 
deflection [7]. An additional benefit of the active suspension is that unlike passive and semi-active suspension systems, it gives dynamic compensation [8].

Active control forces can be implemented by hydraulic, pneumatic or electromagnetic actuators commonly located in parallel with a spring and a damper [9]. Pneumatic actuators are simple, which is their greatest advantage. Hydraulic actuators are well known for their performance in high-power applications. In contrast to hydraulic and pneumatic, accuracy is always much higher in electromagnetic [10]. A new cascade control approach to the active suspension of forest machines using pneumatic actuators and parallel mechanical structure is proposed in [11]. However practically, the force of suspension cannot act directly as the suspension system, thus the suspension force is applied using the hydraulic actuator dynamics in [12]. The electromagnetic actuator replaces the damper and the hydraulic actuator, forming with the spring an oil-free suspension in [13]. A modified energy-saving skyhook for active suspension based on a hybrid electromagnetic actuator is developed in [14]. An active vehicle suspension control approach with electromagnetic and hydraulic actuators is presented in [15].

It is well known that an active damping suspension is an efficient way to enhance the suspension performance and has arduously studied [16]. Various active control strategies are presented based on control methods to improve ride performance. The more effective the control strategy, the better the performance will be. Thus, the selection of control approach for suspension has major impact on the system efficiency. PID controllers are not well suitable as they are incompetent of handling built-in uncertainties [17]. Also, the system robustness reduces with structural uncertainties, so adjusting the gains of PID control law is challenging issue [18]. Many studies concerning active control strategies have been presented during last decades, such as fuzzy logic control [19], $H_{\infty}$ control [20], and backstepping and control [21].

To attain high level of precision, robust control laws are required. Thus, it draws special attention to more advanced active control strategies, for example FOSMC, ISMC, and HOSMC with variant algorithms that can offer an efficient systematic approach to monitor the desired trajectories accurately [22]. LQR is entirely a linear control method whereas FOSMC is a robust control strategy for handling complex systems. Terminal sliding mode control and ISMC are robust techniques of control system, but there is always a discrepancy between the mathematical and physical model, due to mismatched disturbances that cause disparities in the physical model [23-25]. It is well known that FOSMC is insensitive to parameter variations and external disturbances. Whereas, HOSMC is robust against system structure changeability unlike other active control strategies. It reduces harmful oscillation to its maximum level [26]. Hence, HOSMC has all the qualities of SMC and it provides the sliding variable and its derivatives a finite time convergence [27]. Furthermore, the STA algorithm offers finite time stabilization for all the states [28].

In this paper, mathematical model of active suspension system and active model-based dynamic control approaches, i.e., SMC, ISMC, HOSMC are presented. This study deals with theoretical, qualitative and quantitative analysis. The performance of SMC, ISMC and HOSMC are compared with each other, and with conventional LQR, PID, and passive suspension system by using road profile with bump and pothole. It is known that a road with bumps and potholes effect the ride comfort and vehicle stability thus to improve the ride performance, HOSMC based on STA is introduced. Designed controllers are tested by the execution of simulation using MATLAB/Simulink on quarter-vehicle suspension to verify the efficiency of active damping control algorithms in terms of wheel deflection, suspension deflection, and vehicle displacement.

The organization of the paper is as follows: In Section 2, mathematical representation of active suspension quarter-car model is derived. Active control strategies: FOSMC, ISMC, HOSMC, LQR, PID are briefly proposed along with the stability analysis of FOSMC and ISMC in Section 3. The qualitative and quantitative analysis of the proposed controllers along with traditional suspension system are compared and deliberated in Section 4. Lastly, Section 5 executes the conclusion and future work of the paper. 


\section{Mathematical Model of Quarter-Car Active Suspension System}

\subsection{Mathematical Model}

The quarter-car model with active suspension is mostly considered by experts as it holds a simple design. So, it can effectively be expanded to full vehicle model. In Figure 1, quarter-car model is given which is subjected to uneven road surface excitation. In this model, the mass supported by suspension refers to the sprung mass, while wheel and shaft are included in the unsprung mass. Mathematical modeling helps in representing the state-space model of system. The mathematical expression for the quarter-car are obtained by taking the sum of forces acting in the vertical direction on the sprung and unsprung masses. The numerical values of parameters of quarter-car suspension model is presented in Table 1.

Table 1. List of parameters of quarter-car model.

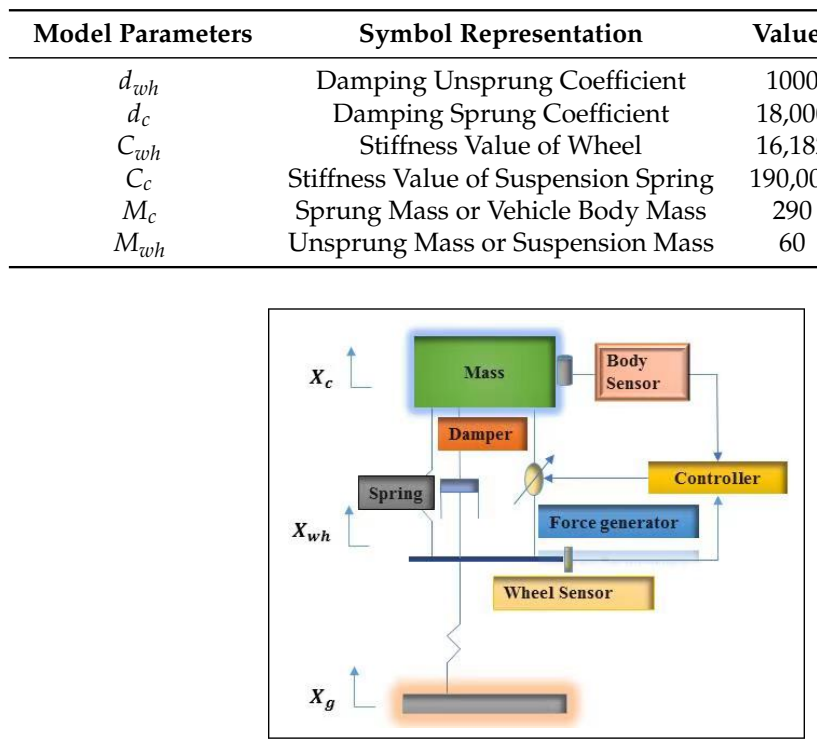

Figure 1. Active suspension system [29].

This model has dynamic characteristics that helps in understanding the system behavior by explaining the correlation between input and output of the system.

Figure 2 represents two-degree of freedom (DOF) quarter-car model, where $X_{c}$ and $X_{w h}$ represent vertical displacement of sprung and unsprung masses. Furthermore, $F(t)$ represents control input and $X_{g}$ is the road surface input of the system.

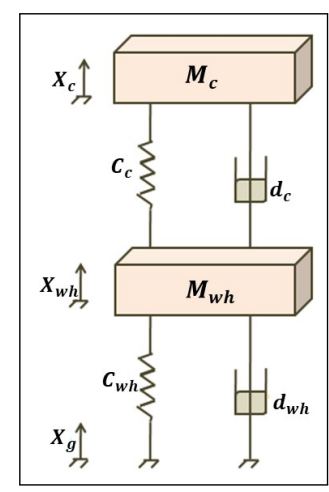

(a)

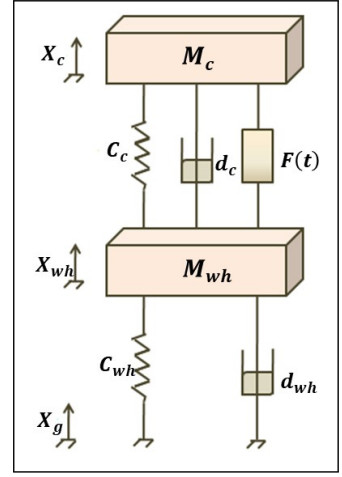

(b)

Figure 2. Suspension models of quarter-car (a) Passive suspension system (b) Active suspension system. 


\subsection{Active Suspension System}

The equation of motion is defined as:

$$
\begin{gathered}
M_{c} \ddot{X}_{c}=-d_{c}\left(\dot{X}_{c}-\dot{X}_{w h}\right)-C_{c}\left(X_{c}-X_{w h}\right)+F(t)+d \\
M_{w h} \ddot{X}_{w h}=d_{c}\left(\dot{X}_{c}-\dot{X}_{w h}\right)-d_{w h}\left(\dot{X}_{w h}-\dot{X}_{g}\right)+C_{c}\left(X_{c}-X_{w h}\right)-C_{w h}\left(X_{w h}-X_{g}\right)-F(t)-d
\end{gathered}
$$

Let us assume that $X, u$ and $u_{d}$ represent the state vector, control input and road disturbances where state variables are as follows:

$$
\begin{aligned}
& X_{1}=X_{c}-X_{w h} \\
& X_{2}=\dot{X}_{c} \\
& X_{3}=X_{w h}-u_{d} \\
& X_{4}=\dot{X}_{w h}
\end{aligned}
$$

$X_{c}-X_{w h}$ and $X_{w h}-u_{d}$ are vehicle suspension travel and wheel deflection. $\dot{X}_{c}$ and $\dot{X}_{w h}$ are vehicle body and wheel velocity respectively. In addition, $\ddot{X}_{c}$ and $\ddot{X}_{w h}$ are vehicle body and wheel acceleration. Hence, in matrix form, we can write:

$$
X=\left[\begin{array}{c}
X_{1} \\
X_{2} \\
X_{3} \\
X_{4}
\end{array}\right]=\left[\begin{array}{c}
X_{c}-X_{w h} \\
\dot{X}_{c} \\
X_{w h}-X_{g} \\
\dot{X}_{w h}
\end{array}\right]
$$

where $u(t)=F(t)$ and $u_{d}=\dot{X}_{g}$. Furthermore, $d$ is bounded external disturbances, i.e., $d \leq|s| \Delta$, where $\Delta>0$. Hence, the equations of motion can be presented in more expedient state-space form:

$$
\dot{X}(t)=A X(t)+B u(t)+E u_{d}+M d
$$

or in matrix form, it can be written as follows:

$$
\left[\begin{array}{c}
\dot{X}_{1} \\
\dot{X}_{2} \\
\dot{X}_{3} \\
\dot{X}_{4}
\end{array}\right]=\left[\begin{array}{cccc}
0 & 1 & 0 & -1 \\
-\frac{C_{c}}{M_{c}} & -\frac{d_{c}}{M_{c}} & 0 & \frac{d_{c}}{M_{c}} \\
0 & 0 & 0 & 1 \\
\frac{C_{c}}{M_{w h}} & \frac{d_{c}}{M_{w h}} & -\frac{C_{w h}}{M_{w h}} & -\frac{d_{c}+d_{w h}}{M_{w h}}
\end{array}\right]\left[\begin{array}{c}
X_{1} \\
X_{2} \\
X_{3} \\
X_{4}
\end{array}\right]+\left[\begin{array}{c}
0 \\
\frac{1}{M_{c}} \\
0 \\
-\frac{1}{M_{w h}}
\end{array}\right] u(t)+\left[\begin{array}{c}
0 \\
0 \\
-1 \\
\frac{d_{w h}}{M_{w h}}
\end{array}\right] u_{d}+\left[\begin{array}{c}
0 \\
1 \\
0 \\
-1
\end{array}\right] d
$$

The model can be extracted as follows:

$$
\begin{aligned}
& \dot{X}_{1}=X_{2}-X_{4} \\
& \dot{X}_{2}=-\frac{C_{c}}{M_{c}} X_{1}-\frac{d_{c}}{M_{c}} X_{2}+\frac{C_{c}}{M_{c}} X_{4}+\frac{1}{M_{c}} u(t)+d \\
& \dot{X}_{3}=X_{4}-u_{d} \\
& \dot{X}_{4}=\frac{C_{c}}{M_{w h}} X_{1}+\frac{d_{c}}{M_{w h}} X_{2}-\frac{C_{w h}}{M_{w h}} X_{3}-\frac{d_{c}}{M_{w h}} X_{4}-\frac{d_{w h}}{M_{w h}} X_{4}-\frac{1}{M_{w h}} u(t)+\frac{d_{w h}}{M_{w h}} u_{d}-d
\end{aligned}
$$

The model presented in (7) has numerous advantages. Due to its simple, and low dynamic order it helps in understanding the dynamic behavior of vehicle. Furthermore, it also provides good basis for designing the active damping controllers. 


\section{Control Methods}

\subsection{PID Controller}

PID controllers are frequently selected for feedback control in automated industry. To measure the resulting error, the PID controller calculates the gap within the measured value of process and optimal set point value. PID has the potential of reducing the steady-state error by regulating the process control inputs.

Figure 3 demonstrates the block diagram of PID controller for the active suspension system. Building blocks of PID controller includes three special variables which in result becomes the reason of the name of controller, i.e., Proportional, Integral and Derivative denoted by $k_{p}, k_{i}$ and $k_{d}$. respectively. These variables are tuning gains of this controller.

$$
u(t)=k_{p} e(t)+k_{i} \int e(t) d t+k_{d} \frac{d e(t)}{d t}
$$

where $e(t)=y_{\text {ref }}-y$ represents the change among the existing value $y$ and relative reference value $y_{\text {ref }}$ of the system.

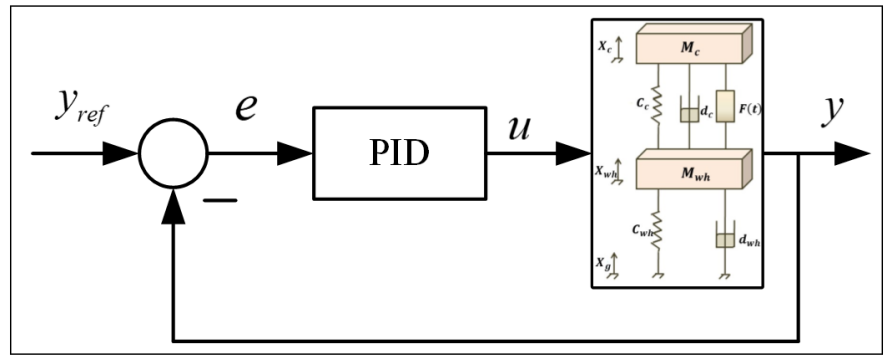

Figure 3. Block diagram of PID controller interface.

\subsection{Linear Quadratic Regulator}

In vehicle suspension control studies, the LQR control scheme is significant. The effectiveness of this technique is that the performance indicator elements are measurable as per the requirements of user or other restraints. The state-variable form of the system is written as follows:

$$
\dot{X}(t)=C x(t)+D u(t)
$$

State and control vector are $x(t) \in R^{n}$ and $u(t) \in R^{m}$, when the initial condition is zero. Here, we suppose that our states are measurable. As state-variable feedback control (SVFB) provides essential closed-loop properties. So, state-variable feedback regulator is written as:

$$
u(t)=-G x(t)
$$

where $G$ is the matrix of feedback gain and $u(t)$ is the control input. Substituting (10) in (9) yields:

$$
\dot{X}(t)=(C-D G) x(t)
$$

The general form of the cost function of the $n$-th order system is written as follows [30]:

$$
M=\int_{0}^{\infty}\left[X^{T}(t) Q(t) X(t)+u^{T}(t) R(t) u(t)\right] d t
$$


To build an optimal SVFB, first we need to define performance index.

$$
M=\frac{1}{2} \int_{0}^{\infty}\left(x(t)^{T} Q x(t)+u(t)^{T} R u(t)\right) d t
$$

where matrix $Q$ and $R$ are representing diagonal matrices of admissible order. Now, replacing the SVFB control in (13), we get:

$$
M=\frac{1}{2} \int_{0}^{\infty} x(t)^{T}\left(Q+G^{T} R G\right) x d t
$$

Therefore, feedback gain $\mathrm{G}$ is represented as follows:

$$
G=R^{-1} D^{T} P
$$

where $P$ is the auxiliary matrix which must satisfy the Riccati equation as given below:

$$
P C+C^{T} P-P D R^{-1} D^{T} P+Q=0
$$

In control theory, Riccati equation has great significance. By using the quadratic equation, we can solve the auxiliary matrix $P$ if the entries of matrices $Q, R, C$ and $D$ are known.

\subsection{First-Order Sliding Mode Control}

FOSMC strategy is acknowledged as one of the efficient solution for vibrant workplace with higher-order systems as it offers robust feedback for nonlinear dynamic systems. In FOSMC design process, performance specifications and closed-loop stability are two primary objectives. Initially, it is essential to find sliding mode surface that enables the system to reach its destined final value. It helps the system to remain unaware of the internal and insignificant disturbances. Final phase is to design feedback control law which directs the controlled variable to its target value. By using sign function, sliding surface is given as follows:

$$
s=\left(\frac{d}{d t}+\lambda\right) e
$$

where $e=y_{r e f}-y$ and $y=X_{c}-X_{w h}$. Now, for the stability condition, consider Lyapunov function as follows:

$$
\begin{gathered}
n=\frac{1}{2} s^{2} \\
\dot{n}=s \dot{s}
\end{gathered}
$$

The time derivative of (17) is derived as follows:

$$
\dot{s}=\ddot{e}+\lambda \dot{e}
$$

By using (7) in (20) yields:

$$
\begin{aligned}
\dot{s}= & \ddot{y}_{r e f}-\dot{X}_{2}+\dot{X}_{4}+\lambda \dot{y}_{r e f}-\lambda\left(X_{2}-X_{4}\right) \\
= & \ddot{y}_{r e f}\left(-\frac{C_{c}}{M_{c}} X_{1}-\frac{d_{c}}{M_{c}} X_{2}+\frac{d_{c}}{M_{c}} X_{4}+d\right)+\left(\frac{C_{c}}{M_{w h}} X_{1}+\frac{d_{c}}{M_{w h}} X_{2}-\frac{C_{w h}}{M_{w h}} X_{3}-\frac{d_{c}}{M_{w h}} X_{4}\right. \\
& \left.+\frac{d_{w h}}{M_{w h}} X_{4}+\frac{1}{M_{w h}} u_{d}-d\right)+\lambda \dot{y}_{r e f}-\lambda\left(X_{2}-X_{4}\right)-\left(\frac{1}{M_{c}}+\frac{1}{M_{w h}}\right) u(t)
\end{aligned}
$$


Using (21) and (17) in (19) we get:

$$
\begin{aligned}
\dot{n}= & s\left[\ddot{y}_{r e f}-\dot{X}_{2}+\dot{X}_{4}+\lambda \dot{y}_{r e f}-\lambda\left(X_{2}-X_{4}\right)\right] \\
= & {\left[\ddot{y}_{r e f}-\left(-\frac{C_{c}}{M_{c}} X_{1}-\frac{d_{c}}{M_{c}} X_{2}+\frac{d_{c}}{M_{c}} X_{4}+d\right)+\left(\frac{C_{c}}{M_{w h}} X_{1}+\frac{d_{c}}{M_{w h}} X_{2}-\frac{C_{w h}}{M_{w h}} X_{3}-\frac{d_{c}}{M_{w h}} X_{4}\right.\right.} \\
& \left.\left.+\frac{d_{w h}}{M_{w h}} X_{4}+\frac{d_{w h}}{M_{w h}} u_{d}-d\right)+\lambda \dot{y}_{r e f}-\lambda\left(X_{2}-X_{4}\right)-\left(\frac{1}{M_{c}}+\frac{1}{M_{w h}}\right) u(t)\right] s
\end{aligned}
$$

Thus, the stability condition can be written as follows:

$$
\dot{n} \leq-\xi|s|
$$

The Lyapunov function should be $V>0$ and $\dot{V} \leq 0$. Initially, the system states will be directed to the sliding mode surface and then hold them on the sliding mode surface as far as the source approaches asymptotically; where $\xi$ is the positive constant. The above expression is known as reaching or sliding condition of the system.

For FOSMC, the standard form of discontinuous control law is given as follows:

$$
U_{D}=k \operatorname{sgn}(s)
$$

where $k$ is constant parameter for manual configuration and is culpable for the reaching part. Chattering phenomena in FOSMC drops the performance of the system which occurs close to the desired equilibrium position. This issue could be fixed more comprehensively if the discontinuous control $U_{D}$ is defined as in [31].

$$
U_{D}=k \frac{s}{s+\delta}
$$

Chattering can be minimized up to significant level through the manual adjustment of the parameter $\delta$, which is the chattering control component. If $e(t)$ is zero, then system can remain on the sliding mode surface all the time. Thus,

$$
\frac{d s}{d t}=0
$$

Feedback control design for SMC consists of two parts, i.e., equivalent control and switching control. Equivalent control $U_{e q}$ is accountable to hold the system trajectories on the sliding surface, while switching/discontinuous control $U_{D}$ is responsible for moving the system trajectories in the direction of sliding surface. $U_{e q}$ can easily be solved when $\dot{s}=0$. Control law for SMC can be expressed as follows:

$$
u(t)=U_{e q}+U_{D}
$$

We can achieve $U_{e q}$ by setting $\dot{s}=0$ in (21) as follows:

$$
U_{e q}=\left[\frac{1}{M_{c}}+\frac{1}{M_{w h}}\right]^{-1}\left(\ddot{y}_{r e f}-f_{1}+f_{2}+\lambda \dot{y}_{r e f}-\lambda\left(X_{2}-X_{4}\right)\right)
$$

where

$$
\begin{aligned}
& f_{1}=-\frac{C_{c}}{M_{c}} X_{1}-\frac{d_{c}}{M_{c}} X_{2}+\frac{d_{c}}{M_{c}} X_{4}+d \\
& f_{2}=\frac{C_{c}}{M_{w h}} X_{1}+\frac{d_{c}}{M_{w h}} X_{2}-\frac{C_{w h}}{M_{w h}} X_{3}-\frac{d_{c}}{M_{w h}} X_{4}-\frac{d_{w h}}{M_{w h}} X_{4}-\frac{1}{M_{w h}} u+\frac{d_{w h}}{M_{w h}} u_{d}-d
\end{aligned}
$$


Now, combining with the discontinuous control while considering the technique of nullifying chattering, the controller can be written as follows:

$$
u(t)=\left[\frac{1}{M_{c}}+\frac{1}{M_{w h}}\right]^{-1}\left(\ddot{y}_{r e f}-f_{1}+f_{2}+\lambda \dot{y}_{r e f}-\lambda\left(X_{2}-X_{4}\right)\right)+k \frac{s}{s+\delta}
$$

\subsection{Integral Sliding Mode Control}

High oscillations in FOSMC harm the performance, decrease the life of actuators and increase the settling time whereas, integral sliding mode minimizes the cost functionality and unwanted oscillations in which system dynamics are weedy against the disturbances. In ISMC, trajectories of the system begin from the sliding surface while the reaching phase is eradicated. The ISMC improves the performance by generalizing higher-order system derivatives and adding trajectories into the sliding surface of the system. It also controls the matched disturbances and sustains the model monotonically. States are moved towards sliding manifolds, and sustainability relies on sliding boundaries. The sliding surface for ISMC can be defined as follows [32]:

$$
s=\dot{e}+\lambda e+z_{i}
$$

where $z_{i}$ is representing an integral part, which helps in termination of the uncertainties of the system. The time derivative of the surface $\dot{s}=0$ to maintain the system on the required path. In this way, the trajectories of the system do not leave the surface $\dot{s}=0$, i.e.,

$$
\dot{s}=\ddot{e}+\lambda \dot{e}+\dot{z}_{i}=0
$$

By using (19) the stability analysis of integral SMC is as follows:

$$
\begin{aligned}
\dot{n} & =s\left(\ddot{e}+\lambda \dot{e}+\dot{z}_{i}\right) \\
& \leq-\xi|s|-\sigma\left(s^{2}\right)
\end{aligned}
$$

where $\sigma$ and $\xi$ are positive factors.

The equivalent controller for ISMC can be obtained as follows:

$$
U_{e q}=\left[\frac{1}{M_{\mathcal{c}}}+\frac{1}{M_{w h}}\right]^{-1}\left(\ddot{y}_{r e f}-f_{1}+f_{2}+\lambda \dot{y}_{r e f}-\lambda\left(X_{2}-X_{4}\right)+z_{i}\right)
$$

Let the total ISMC can be obtained by using Equations (33) and (25) in (27) with invoking the effect of robustness:

$$
u(t)=\left[\frac{1}{M_{c}}+\frac{1}{M_{w h}}\right]^{-1}\left(\ddot{y}_{r e f}-f_{1}+f_{2}+\lambda \dot{y}_{r e f}-\lambda\left(X_{2}-X_{4}\right)+z_{i}\right)+k \frac{s}{s+\delta}
$$

\subsection{High Order Sliding Mode Controller with Super Twisting Algorithm}

To improve the system performance, second-order sliding mode controller based on STA is proposed. When the boundary is known STA generates control function continuously, thus sliding variable and its derivative are driven to zero in predetermined time in the presence of matching uncertainties with bounded gradient. Since the integrand function of STA contains discontinuous function, the chattering is not eliminated but is rather weakened. In contrast to other second-order sliding mode controllers, STA is suitable for controlling the system that appears in the first derivative of the sliding surface $(s)$. 
The physical meaning switching/discontinuous control $U_{D}$ is to make the trajectories of the system to move towards (sliding surface) HOSMC can be expressed as follows:

$$
u(t)=U_{e q}+U_{D}
$$

The equivalent control is obtained by referring to (28)

$$
U_{e q}=\left[\frac{1}{M_{c}}+\frac{1}{M_{w h}}\right]^{-1}\left(\ddot{y}_{r e f}-f_{1}+f_{2}+\lambda \dot{y}_{r e f}-\lambda\left(X_{2}-X_{4}\right)\right)
$$

where

$$
\begin{aligned}
& f_{1}=-\frac{C_{c}}{M_{c}} X_{1}-\frac{d_{c}}{M_{c}} X_{2}+\frac{d_{c}}{M_{c}} X_{4}+d \\
& f_{2}=\frac{C_{c}}{M_{w h}} X_{1}+\frac{d_{c}}{M_{w h}} X_{2}-\frac{C_{w h}}{M_{w h}} X_{3}-\frac{d_{c}}{M_{w h}} X_{4}-\frac{d_{w h}}{M_{w h}} X_{4}-\frac{1}{M_{w h}} u+\frac{d_{w h}}{M_{w h}} u_{d}-d
\end{aligned}
$$

Super twisting algorithm is used for $U_{D}$ representing discontinuous part of the total control law. The STA was introduced as follows [33]:

$$
U_{D}=-k_{1}|s|^{\frac{1}{2}} \operatorname{sgn}(s)+\rho
$$

where $\dot{\rho}$ is defined as follows:

$$
\begin{aligned}
\dot{\rho} & =-k_{2} \operatorname{sgn}(s) \\
& =-k_{2} \operatorname{sgn}\left(\dot{y}_{r e f}-\dot{X}_{1}+\lambda\left(y_{r e f}-X_{1}\right)\right)
\end{aligned}
$$

Here, $k_{1}$ and $k_{2}$ gains are designed and selected as $1.5 \sqrt{\theta}$ and $1.1 \sqrt{\theta}$, respectively, where $\theta$ is the positive constant. This algorithm only works with bounded gradients when the controller is designed to maintain the sliding surface. By using equations of the system, equivalent control (33) and STA for the direct control (38), the control signal of the proposed HOSMC for active quarter-vehicle suspension system is calculated as follows:

$$
\begin{aligned}
u(t)= & {\left[\frac{1}{M_{c}}+\frac{1}{M_{w h}}\right]^{-1}\left(\ddot{y}_{r e f}-f_{1}+f_{2}+\lambda \dot{y}_{r e f}-\lambda\left(X_{2}-X_{4}\right)-k\left|\dot{y}_{r e f}-\dot{X}_{1}+\lambda\left(y_{r e f}-X_{1}\right)\right|^{\frac{1}{2}}\right.} \\
& \operatorname{sgn}\left(\dot{y}_{r e f}-\dot{X}_{1}+\lambda\left(y_{r e f}-X_{1}\right)\right)+\rho
\end{aligned}
$$

If HOSM is properly designed the convergence of HOSMC can be asymptotic and it can totally remove the chattering effect.

\section{Numerical Simulation Results and Discussion}

This section presents numerical simulations results to show the effectiveness of the developed methods. It also demonstrates the impact of various controllers on the system performance. Before illustrating the numerical simulations discussion, a brief description about practical implementation of active vibration control is deliberated. A practical test rig (Quanser) active quarter-car suspension system consists of an amplifier, data acquisition card and a personal computer. The test rig is consisted of three plates led on four columns using linear bearings. The topmost plate is the vehicle body mass $M_{c}$, the middle plate is the wheel axle assembly with mass $M_{w h}$ and the lowest plate is for road inputs driven by a DC motor. There are springs between the plates that present $C_{c}$ and $C_{w h}$. Also, the linear bearings generate damping with $d_{c}$ and $d_{w h}$, respectively. To measure the acceleration of vehicle body an accelerometer is used. Three encoders are used to measure the vehicle body displacement $\dot{X}_{c}$, suspension deflection travel $\left(X_{c}-X_{w h}\right)$, and road input $X_{g}$. A DC motor placed between sprung 
(vehicle body) and unsprung (tire) masses is used to generate desired forces $u(t)$ based on active control laws to overwhelm vibrations of the sprung mass [27].

In this study, simulation based on mathematical model active control algorithms such as PID, LQR, SMC law, ISMC law and HOSMC law are implemented using MATLAB/SIMULINK. Performance of the suspension system in terms of ride performance and vehicle handling is taken into consideration under road disturbances. The main objective is to attain small amplitude value for vehicle body displacement $\dot{X}_{c}$, suspension travel $\left(X_{c}-X_{w h}\right)$ and wheel deflection $\left(X_{w h}-u_{d}\right)$.

Figure 4 shows the block control system of active suspension. The main goal of the controller is to decrease the acceleration and displacement with respect to the open-loop or passive suspension system, to give ride comfort to passengers. The problem is to find the suitable SMC signal $u(t)$ that derives system states to the desired trajectory, i.e., $X(t)=X_{d}$. The controller gain is chosen suitably by hit and trial method until the error $X(t)-X_{d}$ approaches to zero.

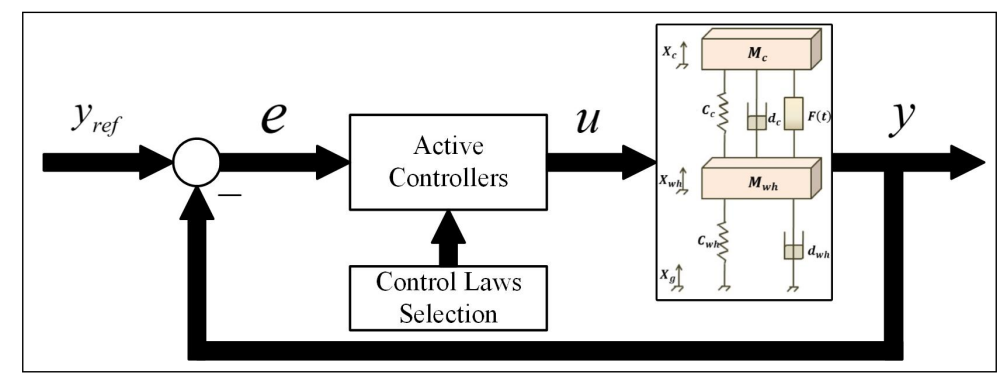

Figure 4. Schematic diagram of the closed-loop control system.

External disturbances $d$ are added to the system as shown in Figure 5.

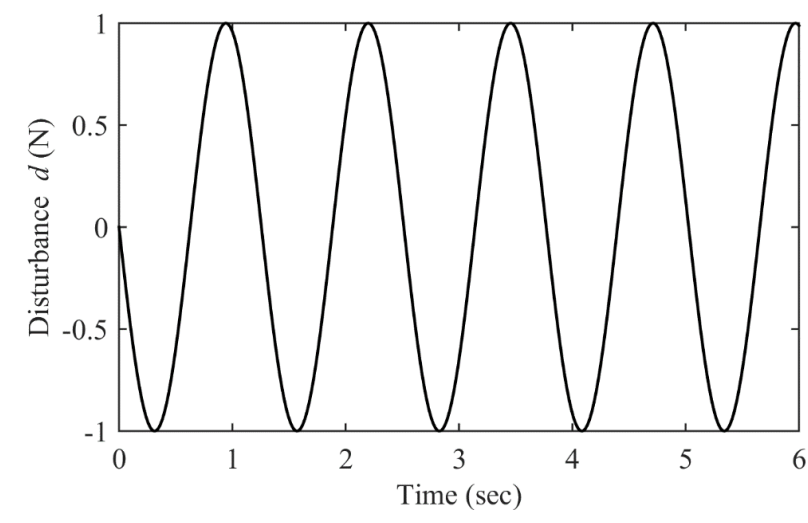

Figure 5. Road disturbances.

Table 2 represents the control parameters for each control strategy.

Table 2. Active controller's parameters.

\begin{tabular}{ccccccccccc}
\hline Active Controllers & $k$ & $\delta$ & $\lambda$ & $k_{1}$ & $k_{2}$ & $Q$ & $R$ & $k_{p}$ & $k_{i}$ & $k_{d}$ \\
\hline HOSMC & - & - & 100 & 31.5 & 2.2 & - & - & - & - & - \\
ISMC & 10 & 0.5 & 50 & - & - & - & - & - & - & - \\
FOSMC & - & - & - & - & - & $\operatorname{diag}(1000,20,80)$ & 1 & - & - & - \\
LQR & 20 & 0.04 & 3 & - & - & - & - & - & - & - \\
PID & - & - & - & - & - & - & - & 100 & 4000 & 50 \\
\hline
\end{tabular}


Figure 6 shows the road disturbances to test the active damping control performances. The simulation time for each simulation is set to $6 \mathrm{~s}$ with frequency 8 HERTZ by following equation.

$$
x_{g}=\left\{\begin{array}{cc}
c(1-\cos 8 \pi t) & 1 \leq t \leq 2 \\
d(1-\cos 8 \pi t) & 3 \leq t \leq 4 \\
0 & \text { otherwise }
\end{array}\right.
$$

where $c=0.03$ and $d=-0.01$.

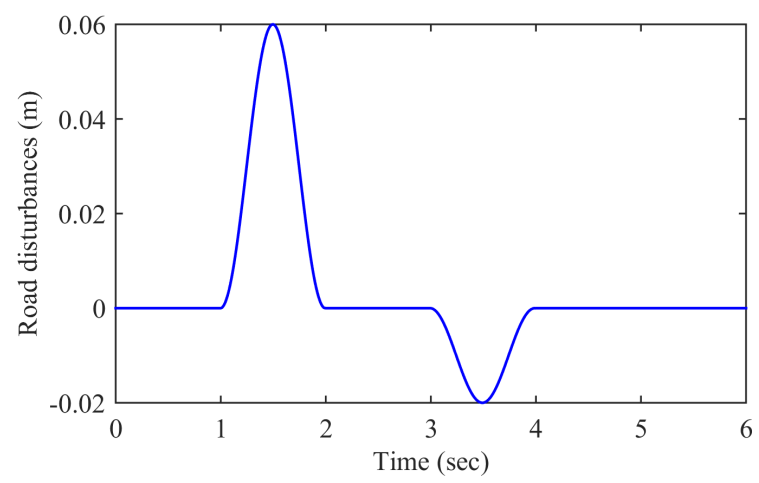

Figure 6. Road Disturbances.

Figures 7 and 8 show the comparison performance of all proposed controllers in terms of vehicle vertical displacement and suspension deflection.

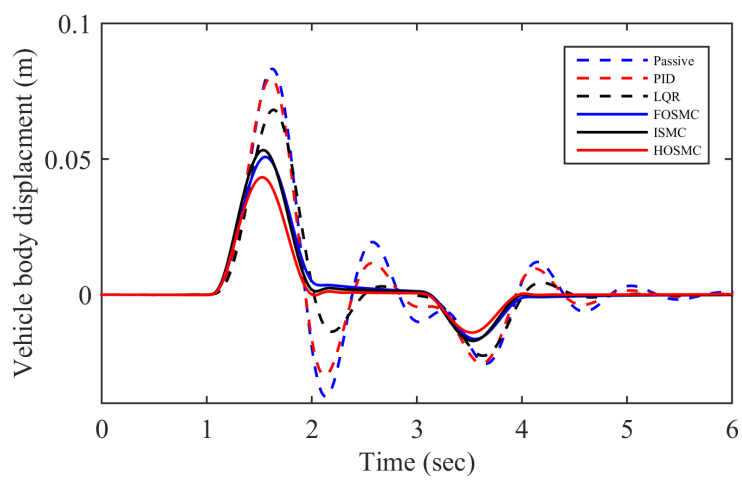

Figure 7. Vehicle body displacement.

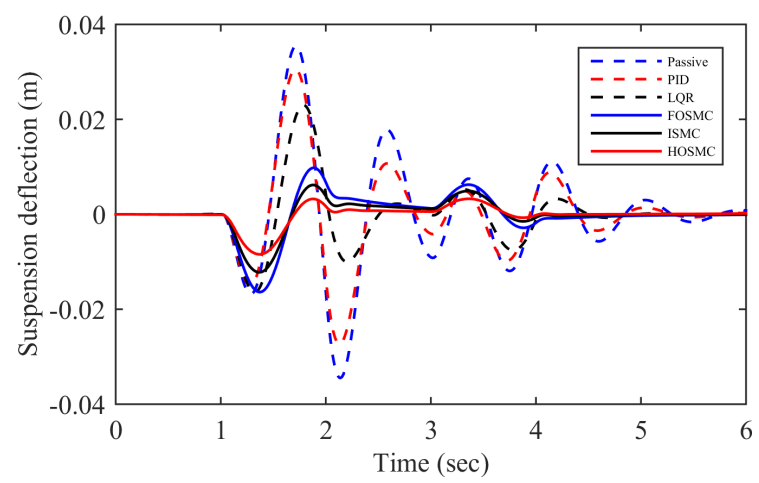

Figure 8. Suspension deflection. 
Moreover, Figure 9 shows the performance comparison analysis of all proposed controllers in terms of wheel deflection of the system.

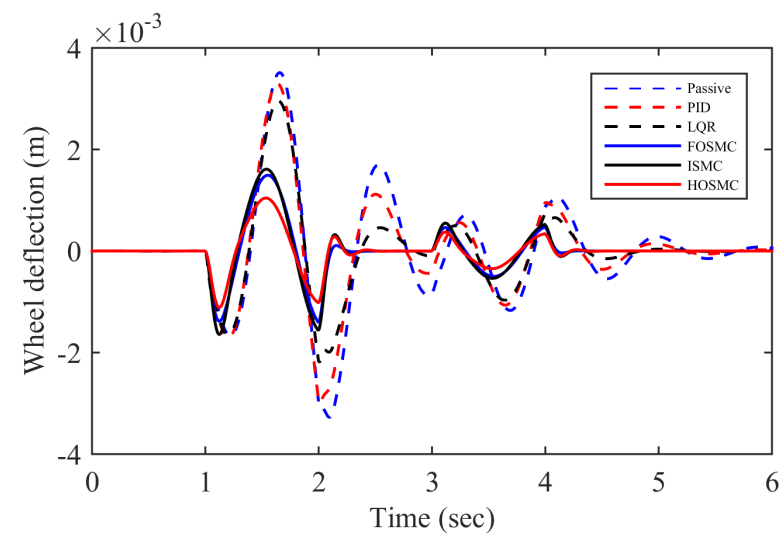

Figure 9. Wheel deflection.

Figure 10 shows the control efforts of each controllers, i.e., PID, LQR, SMC, FOMSC, ISMC and HOSMC. It can be clearly seen that sliding mode control laws produce better performance for active vehicle control. Simulation results prove that PID and LQR give better road holding than passive suspension, while SMC and ISMC achieve better control ride performance. Of all, STA-based HOSMC provides better road holding against road disturbance and also provides superior performance to other active proposed controllers.

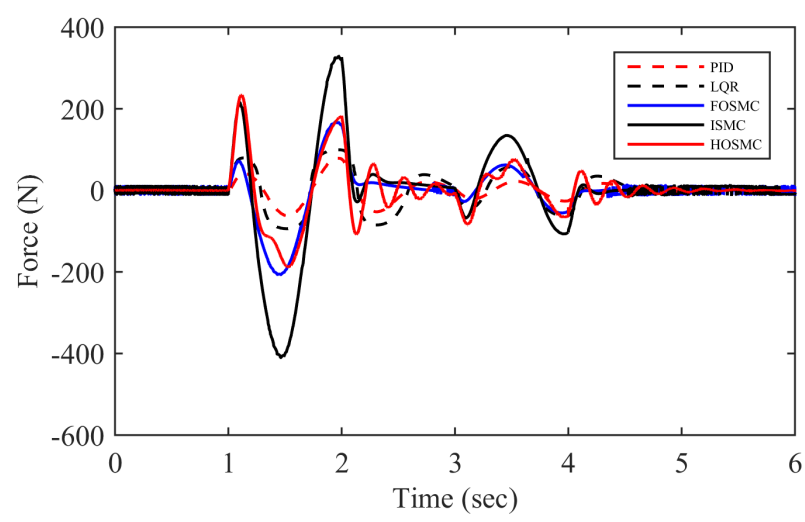

Figure 10. Control Efforts.

It can be clearly noticed that the classical PID and FOSMC performance are superior than passive suspension system and it also reduces the undesired oscillations known as chattering. Although the ride comfort is enhanced by using LQR and ISMC, but the output obtained by STA-based HOSMC yields better performance. When active control signals are applied to the system, the suspension deflection reduces, as seen in the latter figure.

The performance index $(P I)$ which is used to calculate various algorithms is stated as follows:

$$
P I=\frac{1}{2} \int_{0}^{t} H_{p}^{T} Z H_{p} d t
$$


where $H_{p}$ represents displacement vector, $\mathrm{Z}$ is an identity matrix. $H_{p}^{R M S}$ is the root mean square value of displacement sprung and unsprung masses of the system, which are calculated as follows:

$$
\begin{gathered}
H_{p}^{R M S}=\sqrt{\frac{1}{2} \int_{0}^{t}[h(t)]^{2} d t} \\
\ddot{H}_{p}^{R M S}=\sqrt{\frac{1}{2} \int_{0}^{t}[\ddot{h}(t)]^{2} d t}
\end{gathered}
$$

where $h(t)$ and $\ddot{h}(t)$ show the vertical displacement of the sprung mass of the system. The performance indices values of different proposed active controllers are shown in Table 3.

Table 3. Performance indices of different proposed active controllers.

\begin{tabular}{ccccc}
\hline Sprung Mass & Active Controller & $\boldsymbol{P I}$ & $\boldsymbol{H}_{\boldsymbol{p}}^{R M S}$ & $\ddot{\boldsymbol{H}}_{\boldsymbol{p}}^{R M S}$ \\
\hline & Passive & 0.00075 & 0.0222 & 0.7583 \\
& PID & 0.00060 & 0.0209 & 0.6556 \\
\multirow{5}{*}{ Body displacement } & LQR & 0.00054 & 0.01989 & 0.5799 \\
& FOSMC & 0.00043 & 0.01792 & 0.4478 \\
& ISMC & 0.00021 & 0.01298 & 0.3007 \\
& HOSMC & 0.00019 & 0.01156 & 0.2883 \\
\hline
\end{tabular}

Nonlinear control techniques, FOSMC and ISMC have an edge over linear control techniques i.e., PID and LQR. It is evident from the results that STA-HOSMC addresses all the problems and yields better performance as compared to other control algorithms.

\section{Conclusions}

In this paper, active damping control strategies such as FOSMC, ISMC and HOSMC are developed for the quarter-car model to improve the performance for ride ease. The designed sliding mode strategies are compared with LQR, PID and traditional passive suspension system. The performance of each active control is analyzed in terms of vertical displacement, suspension travel and wheel deflection by the execution of simulations using MATLAB/Simulink on the active vehicle suspension system. Also, the performance indices-based performance of controllers are calculated. The quantitative and qualitative analysis verified that the STA-based HOSMC has the ability to diminish the external road disturbances that effects the vehicle stability and provides better control performance to FOSMC, ISMC, PID, LQR and passive suspension system. In future work, this research study can be expanded to design real time optimal nonlinear state feedback control of vehicle suspension to improve the ride ease and vehicle stability.

Author Contributions: Data curation, Y.S.; Investigation, Y.S.; Methodology, Y.S.; Software, Y.S.; Supervision, M.W.; Validation, Y.S.; Writing-original draft, Y.S. All authors have read and agreed to the published version of the manuscript.

Funding: This research was funded by National Natural Science Foundation of China grant number 51775268 and Jiangsu Innovation Program for Graduate Education grant number KYLX16-0328.

Acknowledgments: This work was supported by National Natural Science Foundation of China (Grant No. 51775268), Funding of Jiangsu Innovation Program for Graduate Education (Grant No. KYLX16-0328).

Conflicts of Interest: The authors declare no conflict of interest. 


\section{References}

1. Du, M.; Zhao, D.; Yang, B.; Wang, L. Terminal sliding mode control for full vehicle active suspensionsystems. J. Mech. Sci. Technol. 2018, 32, 2851-2866. [CrossRef]

2. Wang, H.; Mustafa, G.I.; Tian, Y. Model-free fractional-order sliding mode control for an active vehiclesuspension system. Adv. Eng. Softw. 2018, 115, 452-461. [CrossRef]

3. Daniyan, I.; Mpofu, K.; Daniyan, O.; Adeodu, A. Dynamic modelling and simulation of rail car suspension systems using classic controls. Cogent Eng. 2019, 6, 1602927. [CrossRef]

4. Gao, H.; Xue, S.; Yin, S.; Qiu, J.; Wang, C. Output feedback control of multirate sampled-data systems with frequency specifications. IEEE Trans. Control Syst. Technol. 2016, 25, 1599-1608. [CrossRef]

5. Han, S.Y.; Zhong, X.F.; Chen, Y.H.; Tang, G.Y. Discrete approximate optimal vibration control for nonlinear vehicle active suspension. J. Vibro Eng. 2017, 19, 1287-1300. [CrossRef]

6. Harrag, A.; Messalti, S. PSO-based SMC variable step size P \& O MPPT controller for PV systems under fast changing atmospheric conditions. Int. J. Numerical Model. Electronic Netw. Devices Fields 2019, e2603. [CrossRef]

7. M'Sirdi, N.; Rabhi, N.; Fridman, A.; Davila, L.; Delanne, J. Second Order Sliding-Mode Observer for Estimation of Vehicle Dynamic Parameters. Int. J. Veh. Des. 2008, 48, 190-207. [CrossRef]

8. Kashem, S.B.A.; Ektesabi, M.; Nagarajah, R. Comparison between different sets of suspension parameters and introduction of new modified skyhook control strategy incorporating varying road condition. Veh. Syst. Dyn. 2012, 50, 1173-1190 [CrossRef]

9. M'Sirdi, N.; Rabhi, N.; Fridman, A.; Davila, L.; Delanne, J. Load-dependent observer design for active suspension systems. Int. J. Veh. Des. 2015, 68, 163-190.

10. Bai, R.; Guo, D. Sliding-Mode Control of the Active Suspension System with the Dynamics of a Hydraulic Actuator. Complexity 2018, 18, 5907208. [CrossRef]

11. Li, M.; Zhang, Y.; Geng, Y. Fault-tolerant sliding mode control for uncertain active suspension systems against simultaneous actuator and sensor faults via a novel sliding mode observer. Opt. Control Appl. Methods 2018, 39, 1728-1749. [CrossRef]

12. Dong, X.; Zhao, D.; Yang, B.; Han, C. Fractional-order control of active suspension actuator based on parallel adaptive clonal selection algorithm. J. Mech. Sci. Technol. 2016, 30, 2769-2781. [CrossRef]

13. Khan, L.; Qamar, S.; Khan, M.U. Comparative analysis of adaptive NeuroFuzzy control techniques for full car active suspension system. Arabian J. Sci. Eng. 2014, 39, 2045-2069. [CrossRef]

14. Xia, G.; Hua, Y.; Tang, X.; Zhao, L.; Chen, W. Internal-model control of vehicle chassis based on wavelet-network dynamic inversion method. Int. J. Veh. Auton. Syst. 2018, 14, 170-195. [CrossRef]

15. Hasbullah, F.; Faris, W.F. Simulation of disturbance rejection control of half-car active suspension system using active disturbance rejection control with decoupling transformation. J. Phys. Conf. Ser. IOP Publ. 2017, 949, 012025. [CrossRef]

16. Khan, L.; Qamar, S.; Khan, U. 2016. Adaptive PID control scheme for full car suspension control. J. Chin. Ins. Eng. 2016, 39, 169-185. [CrossRef]

17. Huang, Y.; Na, J.; Wu, X.; Gao, G.B.; Guo, Y. Robust adaptive control for vehicle active suspension systems with uncertain dynamics. Trans. Inst. Meas. Control 2018, 40, 1237-1249. [CrossRef]

18. Ikenaga, S.; Lewis, F.L.; Campos, J.; Davis, L. Active suspension control of ground vehicle based on a full-vehicle model. In Proceedings of the 2000 American Control Conference, ACC (IEEE Cat. No. 00CH36334), Chicago, IL, USA, 28-30 June 2000; Volume 6, pp. 4019-4024.

19. Chen, Y.; Wang, Z.L.; Qiu, J.; Huang, H.Z. Hybrid fuzzy skyhook surface control using multi-objective microgenetic algorithm for semi-active vehicle suspension system ride comfort stability analysis. J. Dyn. Syst. Meas. Control 2012, 134, 041003. [CrossRef]

20. Rizvi, S.M.H.; Abid, M.; Khan, A.Q.; Satti, S.G.; Latif, J. $H_{\infty}$ control of 8 degrees of freedom vehicle active suspension system. J. King Saud Univ.-Eng. Sci. 2018, 30, 161-169. [CrossRef]

21. Yagiz, N.; Hacioglu, Y. Backstepping control of a vehicle with active suspensions. Control Eng. Pract. 2008, 16, 1457-1467. [CrossRef]

22. Martins, I.; Esteves, J.; Marques, G.D.; Da Silva, F.P. Permanent-magnets linear actuators applicability in automobile active suspensions. IEEE Trans. Veh. Technol. 2006, 55, 86-94. [CrossRef] 
23. Rosenfield, S. Pros and Cons of Pneumatic, Hydraulic, and Electric Actuation. Motor Control and Switchgear. 2017. Available online: https:/ / electronics360.globalspec.com/article/9480/pros-and-cons-of-pneumatichydraulic-and-electric-actuation (accessed on 8 December 2019).

24. Pujol, G.; Acho, L. Stabilization of the Furuta Pendulum with backlash using H-LMI technique: Experimental validation. Asian J. Control 2010, 12, 460-467.

25. Ding, R.; Wang, R.; Meng, X.; Chen, L. A modified energy-saving skyhook for active suspension based on a hybrid electromagnetic actuator. J. Vib. Control 2019, 25, 286-297. [CrossRef]

26. Beltran-Carbajal, F.; Valderrabano-Gonzalez, A.; Favela-Contreras, A.; Hernandez-Avila, J.L.; Lopez-Garcia, I.; Tapia-Olvera, R. An Active Vehicle Suspension Control Approach with Electromagnetic and Hydraulic Actuators. In Actuators; Multidisciplinary Digital Publishing Institute: Basel, Switzerland, 2019; Volume 8, p. 35.

27. Ozer, H.O.; Hacioglu, Y.; Yagiz, N. High order sliding mode control with estimation for vehicle active suspensions. Trans. Inst. Meas. Control 2018, 40, 1457-1470. [CrossRef]

28. Wang, G.; Chen, C.; Yu, S. Finite-time sliding mode tracking control for active suspension systems via extended super-twisting observer. Proc. Inst. Mech. Eng. Part I J. Syst. Control Eng. 2018, 231, 459-470. [CrossRef]

29. Levant, A. Sliding order and sliding accuracy in sliding mode control. Int. J. Control 1993, 58, 1247-1263. [CrossRef]

30. Levant, A. Principles of 2-sliding mode design. Automatica 2007, 43, 576-586. [CrossRef]

31. Qamar, S.; Khan, L.; Ali, S. Adaptive B-spline based neuro-fuzzy control for full car active suspension system. Middle-East J. Sci. Res. 2013, 16, 1348-1360.

32. Irfan, S.; Mehmood, A.; Razzaq, M.T.; Iqbal, J. Advanced sliding mode control techniques for inverted pendulum: Modelling and simulation. Eng. Sci. Technol. Int. J. 2018, 21, 753-759. [CrossRef]

33. Youness, S.; Lobusov, E. Networked control for active suspension system. Procedia Comput. Sci. 2019, 150, 123-130. [CrossRef]

(C) 2019 by the authors. Licensee MDPI, Basel, Switzerland. This article is an open access article distributed under the terms and conditions of the Creative Commons Attribution (CC BY) license (http:/ / creativecommons.org/licenses/by/4.0/). 